\title{
COURBURES SCALAIRES DES VARIÉTÉS D'INVARIANT CONFORME NÉGATIF
}

\author{
ANTOINE RAUZY
}

\begin{abstract}
In this paper, we are interested in the problem of prescribing the scalar curvature on a compact riemannian manifold of negative conformal invariant. We give a necessary and sufficient condition when the prescribed function $f$ is nonpositive. When $\sup (f)>0$, we merely find a sufficient condition. This is the subject of the first theorem. In the second one, we prove the multiplicity of the solutions of subcritical (for the Sobolev imbeddings) elliptic equations. In another article [8], we will prove the multiplicity of the solutions of the prescribing curvature problem, i.e. for a critical elliptic equation.
\end{abstract}

Cet article est le premier d'une série de deux; on donne ici la démonstration de résultats parus dans la note à l'Académie des Sciences [7]. On s'intéresse au problème de la courbure scalaire prescrite dans le cas d'une variété riemannienne d'invariant conforme négatif. On démontre une condition nécessaire et suffisante pour que le probleme admette une solution lorsque la fonction prescrite $f$ est négative ou nulle. Lorsque $\sup f>0$, on n'obtient plus que des conditions suffisantes (même si l'on démontre que l'une d'entre elles est nécessaire). Ces résultats font l'objet du Théorème 1. Un second théorème prouve la multiplicité des solutions d'équations elliptiques sous-critiques (relativement aux inclusions de Sobolev).

Dans le second article [8], on s'intéressera à la multiplicité des solutions du problème de courbure prescrite qui conduit à une équation elliptique dont l'exposant est, cette fois, critique.

\section{POSITION DU PROBLEME}

Soit $(M, g)$ une variété riemannienne de dimension $n, n \geq 3$. On suppose $M$ compacte et telle que l'invariant conforme $\mu$ soit négatif

$$
\mu=\inf _{u \neq 0, u \geq 0} \frac{\frac{4(n-1)}{n-2} \int_{M}|\nabla u|^{2}+\int_{M} R u^{2}}{\left[\int_{M} u^{2 n /(n-2)}\right]^{(n-2) / n}}
$$

où $R$ est la courbure scalaire de $M$. On considère le problème suivant: étant donnée une fonction $f$ continue sur $M$, existe-t-il une transformation conforme de la métrique $g \rightarrow g^{\prime}=u^{4 /(n-2)} g, u>0$, telle que $f$ soit la courbure scalaire de la variété $\left(M, g^{\prime}\right)$ ?

Received by the editors March 30, 1994.

1991 Mathematics Subject Classification. Primary 53C42; Secondary 58G03. 
A une transformation conforme près (de la métrique), puisque $\mu$ est négatif, on peut toujours considérer $(M, g)$ comme une variété de courbure scalaire constante $R<0$ et de volume 1 , ce que l'on fera dorénavant.

Analytiquement on cherche une solution de l'équation

$$
\Delta u+\frac{n-2}{4(n-1)} R u=\frac{n-2}{4(n-1)} f u^{(n+2) /(n-2)} \text { où } u>0 .
$$

On s'intéresse au cas particulier où $f$ change de signe sur $M$. En effet, si $f$ est une constante négative, l'équation (1) admet une solution constante évidente et Aubin [3, p. 135] a montré que cette solution était unique. Le cas $f<0$ a été entièrement traité par Aubin [2] par une méthode variationnelle, il a montré que si $f<0$ sur $M$, l'équation (1) admet une unique solution. Kazdan-Warner [4] et [5] ont retrouvé ce résultat par une méthode de sur et sous-solutions. Cependant ces deux démonstrations ne peuvent pas être utilisées lorsque $f$ s'annule ou change de signe.

Kazdan et Warner [4] ont prouvé d'autre part trois propositions qui s'appliquent dans le cas géneŕal:

1. Pour que l'équation (1) admette une solution, il est nécessaire que $\int_{M} f$ soit strictement négative.

2. Il existe des fonctions $f C^{\infty}$ sur $M$ qui satisfont $\int_{M} f<0$, sans que l'équation (1) n'admette de solution.

3. Si l'équation (1) admet une solution pour une fonction $f$, elle admet aussi une solution pour toute fonction $f^{\prime}$ vérifiant $f^{\prime} \leq f$.

Dans tout ce qui suit on supposera donc $\int_{M} f<0$.

Lorsque $f$ s'annule ou change de signe, la situation peut être très différente du cas $f<0$. Dans ce cas, les auteurs qui se sont intéressés au problème s'écartent de la question géométrique en cherchant pour quelles valeurs de $\lambda$, l'équation

$$
\Delta u-\lambda u=f u^{(n+2) /(n-2)}
$$

admet une solution positive ( $f$ étant une fonction $C^{\infty}$ sur $M$, qui s'annule ou change de signe).

Les premiers, Kazdan et Warner ont montré que si $f$ est strictement positive en au moins un point, alors il existe une valeur maximale $\lambda_{0}$ de $\lambda$ telle que:

si $0<\lambda<\lambda_{0},\left(E_{\lambda}\right)$ admet une solution,

si $\lambda>\lambda_{0},\left(E_{\lambda}\right)$ n'a pas de solution.

Ouyang [6], Vazquez et Véron [9] ont montré que cette valeur limite existait aussi si le maximum de $f$ est nul. Cette étude analytique de l'équation $\left(E_{\lambda}\right)$ se traduit pour le problème géométrique par les résultats suivants pour l'équation (1):

Pour $f$ donnée, on pose

$$
M_{1}=\{x \in M / f(x) \geq 0\},
$$

$V\left(M_{1}\right)$ : l'ensemble des variétés à bord incluses dans $M_{1}$, on suppose $V\left(M_{1}\right)$ non vide. On définit la grandeur géométrique $\lambda_{1}=\inf _{\Omega} \lambda_{\Omega}$ où $\lambda_{\Omega}$ est la première valeur propre du laplacien pour le problème de Dirichlet associé à $\Omega, \Omega$ parcourant $V\left(M_{1}\right)$. Si $M_{1}$ est lui-même une variété à bord, $\lambda_{1}=\lambda_{M_{1}}$. Vazquez et Véron ont montré le théorème suivant obtenu pour une fonction $f$ qui s'annule sans changer de signe: 
Théorème.

$S i|R|<\frac{4(n-1)}{n-2} \lambda_{1}$, l'équation (1) admet une unique solution non triviale.

Si $M_{1}$ est une variété à bord, on a le résultat complet suivant:

si $|R|<\frac{4(n-1)}{n-2} \lambda_{1}$, l'équation (1) admet une unique solution non triviale.

si $|R| \geq \frac{4(n-1)}{n-2} \lambda_{1}$, l'équation (1) n'admet pas de solution non identiquement nulle.

Lorsque $f$ est strictement positive en un point, on n'a plus que le résultat suivant:

Théorème. Soit $f C^{\infty}$ sur $M, f>0$ en un point de $M$, alors il existe $\lambda_{0} \geq \lambda_{1}$ tel que si $|R|>\frac{4(n-1)}{n-2} \lambda_{0}$, l'équation (1) n'admet pas de solution non triviale.

Mentionnons enfin que T. Ouyang énonce le théorème suivant (dont la démonstration n'a pas paru).

Soit $\lambda_{0}$ le maximum des valeurs de $\lambda$ tel que l'équation $\left(E_{\lambda}\right)$ admette une solution. Si sup $f>0$ et $\lambda<\lambda_{0},\left(E_{\lambda}\right)$ admet plusieurs solutions. Elle n'en admet qu'une lorsque $\lambda=\lambda_{0}$. Si sup $f=0$, l'équation $\left(E_{\lambda_{0}}\right)$ n'admet pas de solution non triviale.

Ainsi, lorsque $f$ change de signe on ne dispose plus que de conditions nécessaires à l'existence de solutions de l'équation (1). On est donc amené à rechercher des conditions suffisantes sur $f$. Dans ce but, on utilise une méthode variationnelle du type de celle de Yamabe. On s'aperçoit cependant que la fonctionnelle classique

$$
\frac{\frac{4(n-1)}{n-2} \int_{M}|\nabla u|^{2}+\int_{M} R u^{2}}{\left[\int_{M} f u^{2 n /(n-2)}\right]^{(n-2) / n}}
$$

ne convient pas ici, et qu'il est préférable de la modifier quelque peu. En effet cette fonctionnelle n'est pas minorée a priori. Lorsqu'on impose des contraintes de façon à obtenir un minimum, les multiplicateurs de Lagrange que l'on introduit sont difficiles à contrôler.

Par cette méthode, on trouve des conditions suffisantes pour que l'équation (1) admette des solutions lorsque $f$ change de signe, de plus lorsque $f$ s'annule sans changer de signe ces conditions sont nécessaires. La démonstration permet de retrouver les résultats d'existence de Kazdan et Warner ainsi que ceux de Ouyang et Vazquez-Véron.

Par ailleurs on prouve que sous certaines hypothèses, l'équation

$$
\Delta u+\frac{n-2}{4(n-1)} R u=\frac{n-2}{4(n-1)} f u^{q-1},
$$

où $q$ est inférieur à l'exposant critique de Sobolev $\frac{2 n}{n-2}$, admet au moins deux solutions.

Notations. On pose:

(a) $\widetilde{R}=\frac{n-2}{4(n-1)} R, \widetilde{R}<0$.

(b) $N=\frac{2 n}{n-2}, n$ dimension de la variété.

(c) \|\|$_{p}$ désigne la norme dans $L_{p} ; H_{1}^{2}$ est l'espace de Sobolev des fonctions de $L_{2}$, dont le gradient est dans $L_{2}$.

(d) $K_{1}$ et $A_{1}$ sont deux constantes telles que $\forall u \in H_{1}^{2}(M)$, on ait: 


$$
\|u\|_{N}^{2} \leq K_{1}\|\nabla u\|_{2}^{2}+A_{1}\|u\|_{2}^{2} \text {. }
$$

(e) $f^{-}=\inf (f, 0)$ et $f^{+}=\sup (f, 0)$.

\section{ENONCÉ DES RÉSULTATS}

Soit $f$ une fonction $C^{\infty}$ sur $M$. On définit

$$
\lambda_{f}=\inf _{u \in \mathscr{A}} \frac{\int|\nabla u|^{2}}{\int|u|^{2}}
$$

avec $\mathscr{A}=\left\{u \in H_{1}^{2}, u \geq 0, u \neq 0\right.$ telle que $\left.\int_{M}\left|f^{-}\right| u=0\right\}$. $\lambda_{f} \in \overline{\mathbb{R}}$, et si $\mathscr{A}=\varnothing$, on pose $\lambda_{f}=+\infty$.

II.1. Equation critique.

Théorème 1. Il existe une constante $C$ strictement positive, qui ne dépend que de $f^{-} / \int f^{-}$, telle que si $f C^{\infty}$ sur $M$ vérifie les conditions suivantes:

$$
\begin{gathered}
|R|<\frac{4(n-1)}{n-2} \lambda_{f}, \\
\quad \frac{\left(\sup f^{+}\right)}{\int\left|f^{-}\right|}<C,
\end{gathered}
$$

l'équation (1) admet une solution partout positive. $f$ est alors la courbure scalaire d'une métrique conforme à $g$. La condition (1) est nécessaire.

Remarque 1. La condition (1): $|R|<\frac{4(n-1)}{n-2} \lambda_{f}$ est toujours nécessaire. Dans le cas particulier où $f$ s'annule sans changer de signe, c'est-à-dire $f^{+} \equiv 0$, la condition (2) est toujours vérifiée, la condition (1) devient donc nécessaire et suffisante.

Remarque 2. On retrouve les conditions d'existence de solutions de Kazdan et Warner, et de Vazquez-Véron. On a en effet le résultat suivant:

Lemme 1. Pour une fonction $f C^{\infty}$ telle que $M_{1}=\{x \in M / f(x) \geq 0\}$ soit une variété à bord, les deux réels $\lambda_{1}$, première valeur propre du laplacien du problème de Dirichlet associé à $M_{1}$, et $\lambda_{f}$, introduit ci-dessus, coïncident.

De plus on verra qu'il est naturel de poser $\lambda_{f}=+\infty$ lorsque $M_{1}=$ $\{x \in M / f(x) \geq 0\}$ est de mesure nulle. Ainsi pour $f \leq 0, \sup f^{+}=0$ et la condition (2) du théorème est vérifiée; pour $f<0, \lambda_{f}=+\infty$ et la condition (1) est vérifiée. Les théorèmes d'existence précédents sont compris dans l'énoncé du théorème 1 .

Remarque 3. On obtient immédiatement des exemples de fonctions $f C^{\infty}$ permettant d'obtenir des solutions en remarquant que

$$
\int_{f \geq 0} 1<\left[A_{1}+K_{1} \frac{n-2}{4(n-1)}|R|\right]^{-n / 2} \quad \text { implique } \quad \lambda_{f}>\frac{n-2}{4(n-1)}|R| .
$$

(Voir lemme 2.) $A_{1}$ et $K_{1}$ sont les constantes des inégalités de Sobolev.

II.2. Equation sous-critique. Si l'exposant de l'équation (2) n'est pas critique, c'est-à-dire lorsque $q \in] 2, \frac{2 n}{n-2}[$, on obtient le théorème suivant: 
Théorème 2. Pour toute fonction $f C^{\infty}$ sur $M$, il existe une constante $C_{q}$ strictement positive, qui ne dépend que de $f^{-} / \int f^{-}$, telle que si $f$ vérifie les conditions suivantes:

$$
\begin{aligned}
& \text { (1) }|R|<\frac{4(n-1)}{n-2} \lambda_{f}, \\
& \text { (2) }\left(\sup f^{+}\right) / \int\left|f^{-}\right|<C_{q}, \\
& \text { (3) } \sup f>0,
\end{aligned}
$$

alors l'équation (2) admet deux solutions distinctes non identiquement nulles.

Pour un exposant $q$ non critique, on peut résumer la situation ainsi: on cherche à minimiser une fonctionnelle $F_{q}$ pour des fonctions de norme fixée dans $L_{q}$. Soit $k$ cette norme, $\mu_{k, q}$ le minimum.

$$
\left.F_{q}(u)=\int|\nabla u|^{2}+\widetilde{R} \int u^{2}-\int f u^{q} \quad \text { où } q \in\right] 2, N[,
$$

$u$ est une fonction qui appartient à l'ensemble

$$
\mathscr{B}_{k, q}=\left\{u \in H_{1}^{2}, u \geq 0,\|u\|_{q}^{q}=k\right\}
$$

et on note $\mu_{k, q}=\inf _{u \in \mathscr{F}_{k, q}} F_{q}(u)$.

On obtient trois types de courbes $\mu_{k, q}(k)$ :

Le premier cas correspond à une fonction $f$ telle que $f<0$, ou $f \leq 0$ et $\int_{f=0} 1=0$, ou $f \leq 0$ et $\lambda_{f}>\frac{n-2}{4(n-1)}|R|$, la courbe $k \rightarrow \mu_{k, q}(k)$ part de 0 , décroît passe par un minimum négatif puis croît et tend vers $+\infty$ avec $k$.

Le deuxième type correspond à des fonctions telles que sup $f>0$. Cette fois la courbe $k \rightarrow \mu_{k, q}(k)$ part de 0 , décroît passe par un minimum négatif puis croît passe par un maximum et finalement tend vers $-\infty$ avec $k$. On montre ici que dans le cas où ce maximum est positif on obtient deux solutions pour l'équation (2).

Le troisième type, dont on ne montre pas l'existence, représente les cas où l'on ne peut pas minimiser la fonctionnelle. La courbe $k \rightarrow \mu_{k, q}(k)$ part de 0 , ne cesse de décroître et tend vers $-\infty$ avec $k$. On ne peut donc rien conclure par la méthode utilisée ici.

\section{ETUde DE $\lambda_{f}$. Condition NECesSAire}

III.1. Démonstration du lemme 1. Le lemme 1 énonçait: pour une fonction $f C^{\infty}$ telle que $M_{1}=\{x \in M / f(x) \geq 0\}$ soit une variété à bord, les deux réels $\lambda_{1}$ et $\lambda_{f}$, coïncident. Supposons donc que $M_{1}$ soit une variété à bord. On a $\lambda_{f}=\inf _{u \in \mathscr{A}} \int|\nabla u|^{2} / \int|u|^{2}$. Avec $\mathscr{A}=\left\{u \in H_{1}^{2}, u \geq 0, u \neq 0\right.$ telle que $\left.\int_{M}\left|f^{-}\right| u=0\right\} . \lambda_{1}$, tel qu'il est introduit par Ouyang et Véron, est défini par

$$
\lambda_{1}=\inf _{u \in \mathscr{A}^{\prime}} \frac{\int_{M_{1}}|\nabla u|^{2}}{\int_{M_{1}}|u|^{2}}
$$

avec $\mathscr{A}^{\prime}=\left\{u \in C^{\infty}, u>0\right.$ sur $M_{1}, u=0$ sur $\left.\partial M_{1}\right\}$.

Immédiatement on a l'inclusion de $\mathscr{A}^{\prime}$ dans $\mathscr{A}$, donc $\lambda_{f} \leq \lambda_{1}$. Or une étude classique montre que $\lambda_{f}$ est atteint, et d'après les théorèmes de régularité, 
une fonction qui réalise ce minimum est $C^{\infty}$ sur $M_{1}$. Il existe donc $u_{0}$ continue telle que $u_{0} \geq 0, u_{0} \not \equiv 0$ et

Donc $u_{0} \in \mathscr{A}^{\prime}$ et $\lambda_{f}=\lambda_{1}$.

$$
\int_{M}\left|f^{-}\right| u_{0}=0 \text {, qui vérifie } \frac{\int_{M}\left|\nabla u_{0}\right|^{2}}{\int_{M}\left|u_{0}\right|^{2}}=\lambda_{f} \text {. }
$$

III.2. Estimation de $\lambda_{f}$. De la définition de $\lambda_{f}$, on déduit deux propriétés réunies ici sous la forme d'un lemme:

Lemme 2. (1) Pour tout $\varepsilon$ donné, il existe des fonctions $f$ telles que $\lambda_{f}<\varepsilon$.

(2) Lorsque $\int_{f \geq 0} 1$ tend vers zéro ( $f$ étant considérée ici comme variable), $\lambda_{f}$ tend vers l'infini. En particulier la condition

$$
\int_{f \geq 0} 1 \leq\left[A_{1}+K_{1} \frac{n-2}{4(n-1)}|R|\right]^{-n / 2} \text { implique } \lambda_{f}>|\widetilde{R}| \text {. }
$$

Démonstration. (1) Considérons une fonction $f_{\eta}$ strictement négative sur une boule de centre $x_{0}$, de rayon $\eta, B\left(x_{0}, \eta\right), f_{\eta}$ étant positive ou nulle en dehors de cette boule. Soit $u_{\eta}$ une fonction radiale qui vaut 1 à l'extérieur de la boule de centre $x_{0}$, de rayon $2 \eta$, zéro à l'intérieur de $B\left(x_{0}, \eta\right), u_{\eta}$ étant continue et affine pour $r \in[\eta, 2 \eta]$. Alors $\lambda_{f_{\eta}} \leq \int_{M}\left|\nabla u_{\eta}\right|^{2} / \int_{M}\left|u_{\eta}\right|^{2}$. Comme les coefficients de la métrique sont bornés, il existe deux constantes positives $c$ et $c^{\prime}$ telles que, $\eta$ étant une constante positive:

$$
\lambda_{f_{n}} \leq c \frac{\int_{\eta}^{2 \eta} \frac{1}{\eta^{2}} r^{n-1} d r}{1-\eta^{n} c^{\prime}} \leq c \frac{2^{n}-1}{n} \frac{\eta^{n-2}}{1-\eta^{n} c^{\prime}} .
$$

Ainsi $\lambda_{f_{\eta}}$ tend vers zéro avec $\eta$.

(2) Pour montrer la deuxième propriété on utilise l'inégalité de Hölder

$$
\int_{f \geq 0}|u|^{2} \leq\left[\int_{f \geq 0} u^{N}\right]^{2 / N}\left[\int_{f \geq 0} 1\right]^{(N-2) / N} \text {. }
$$

Considérons des fonctions de $\mathscr{A}$ d'après l'inégalité de Sobolev

$$
\begin{gathered}
\|u\|_{N}^{2} \leq K_{1}\|\nabla u\|_{2}^{2}+A_{1}\|u\|_{2}^{2} . \\
\int_{f \geq 0}|u|^{2} \leq\left[\int_{f \geq 0} 1\right]^{(N-2) / N}\left(K_{1}\|\nabla u\|_{2}^{2}+A_{1}\|u\|_{2}^{2}\right) . \text { Ainsi puisque } \frac{N-2}{N}=\frac{2}{n} \\
\lambda_{f} \geq \frac{1}{K_{1}}\left[\left(\int_{f \geq 0} 1\right)^{-2 / n}-A_{1}\right] .
\end{gathered}
$$

Donc, lorsque $\int_{f \geq 0} 1$ tend vers zéro, $\lambda_{f}$ tend vers l'infini, on en déduit

$$
\int_{f \geq 0} 1 \leq\left[A_{1}+K_{1} \frac{n-2}{4(n-1)}|R|\right]^{-n / 2} \text { implique } \lambda_{f}>|\widetilde{R}| .
$$

De cette propriété et du théorème 1 , on déduit le lemme suivant:

Lemme 3. Dans la classe conforme d'une métrique à courbure scalaire constante négative, il existe toujours une métrique dont la courbure scalaire change de signe.

III.3. Condition nécessaire. Soit $M_{1}=\{x \in M / f(x) \geq 0\}$; si $M_{1}=\varnothing$, $\lambda_{f}=+\infty$, la condition est inutile. Si $M_{1}=M, \lambda_{f}=0$ et l'équation (1) n'a effectivement pas de solution. 
Si $M_{1} \neq \varnothing$, soit $\Omega$ un ouvert à bord $C^{\infty}$ tel que $M_{1} \subset \Omega$, soit $\Omega^{\prime}$ un ouvert à bord $C^{\infty}$ vérifiant $M_{1} \subset \Omega^{\prime} \subset \Omega$ et $\partial \Omega^{\prime} \cap \partial \Omega=\varnothing$. Soit $\eta$ une fonction plateau qui vaut 1 sur $\Omega^{\prime}$, à support dans $\Omega$ et $u$ une fonction de $\mathscr{A}$, alors $\eta u \in \mathscr{A}$, mais aussi $\eta u \in \stackrel{\circ}{1}_{1}^{2}(\Omega)$. On a donc $\|\nabla \eta u\|_{2}^{2}=\int|\nabla \eta|^{2} u^{2}+$ $2 \int(\nabla \eta \cdot \nabla u) \eta u+\int|\nabla u|^{2} \eta^{2}$. Or $\nabla \eta$ est borné et vaut 0 sur $\Omega^{\prime}$ et $u=0$ presque partout sur $M \backslash \Omega^{\prime}$ donc $\int|\nabla \eta|^{2} u^{2}=0$ et $\int(\nabla \eta \cdot \nabla u) \eta u=0$ car $\eta u \nabla \eta=0$ presque partout sur $M$ et $\nabla u \in L^{2}$. Mais par ailleurs $\|\eta u\|_{2}^{2}=\|u\|_{2}^{2}$ donc $\lambda_{f}=\inf _{u \in \mathscr{B}(\Omega)} \int|\nabla u|^{2} / \int|u|^{2}$, avec $\mathscr{B}(\Omega)=\left\{u \in \stackrel{\circ}{H}_{1}^{2}(\Omega), u \geq 0, u \not \equiv 0\right.$ telle que $\left.\int_{M}\left|f^{-}\right| u=0\right\}$.

Si on considère alors $\Omega_{j}$ une suite d'ouverts emboîtés vérifiant

(1) $\Omega_{j}$ est un ouvert à bord $C^{\infty}$ contenant $M_{1}$,

(2) $\forall x \in M$ tel que $f(x)<0$, il existe $J$ tel que $x \in \Omega_{J}$,

on peut définir en reprenant les notations de Vazquez-Véron:

$$
\lambda_{\Omega_{j}}=\inf _{u \in \mathscr{Q}_{j}} \int|\nabla u|^{2} / \int|u|^{2}, \text { avec } \mathscr{A}_{j}=\left\{u \in \stackrel{\circ}{H}_{1}^{2}\left(\Omega_{j}\right), u \geq 0,\|u\|_{2}^{2}=1\right\} .
$$

On a $\mathscr{B}\left(\Omega_{j}\right) \subset \mathscr{A}_{j}$ donc $\lambda_{f} \geq \lambda_{\Omega_{j}}$.

Par conséquent, si $\forall j, v_{j} \geq 0$ est une fonction propre du laplacien pour le problème de Dirichlet associé à $\Omega_{j}$ de norme $L^{2}$ égale à 1 , les $v_{j}$ forment une suite bornée de $H_{1}^{2}(M)$, donc à une sous-suite près convergent faiblement vers une fonction $v$ lorsque $j$ tend vers l'infini. Les $v_{j}$ convergent aussi fortement dans $L^{2}$ et presque partout vers la même fonction $v$ d'où $\lim _{j \rightarrow \infty} \lambda_{\Omega_{j}} \geq \int|\nabla v|^{2}$. D'autre part pour tout $x$ de $M$ tel que $f(x)<0$, $v(x)=0$. On en déduit $v \in \mathscr{A}$ et $\lim _{j \rightarrow \infty} \lambda_{\Omega_{j}}=\lambda_{f}$.

Il est alors facile de montrer $|\widetilde{R}| \leq \lambda_{f}$. En effet

$$
\int_{\Omega_{j}} \Delta v_{j} u-\int_{\Omega_{j}} \Delta u v_{j}=-\int_{\partial \Omega_{j}} \frac{\partial v_{j}}{\partial n} u \geq 0
$$

donc

$$
\left(\lambda_{\Omega_{j}}+\tilde{R}\right) \int_{\Omega_{j}} v_{j} u-\int_{\Omega_{j}} f u^{N-1} v_{j} \geq \inf _{M} u \int_{\partial \Omega_{j}}-\frac{\partial v_{j}}{\partial n}>0
$$

puisque

$$
\int_{\Omega_{j}} \Delta v_{j}=\int_{\partial \Omega_{j}}-\frac{\partial v_{j}}{\partial n}
$$

Comme lorsque $j$ tend vers l'infini $\sup _{\Omega_{j}}(f)<\varepsilon$ pour tout $\varepsilon>0, \lim \lambda_{\Omega_{j}}+\widetilde{R} \geq$ 0 et donc $\lambda_{f} \geq|\widetilde{R}|$.

Montrons alors l'inégalité stricte $\left(\lambda_{f} \neq|\widetilde{R}|\right)$.

$$
\left|\lambda_{\Omega_{j}}+\widetilde{R}\right| \sup _{M} u \int_{\Omega_{j}} v_{j}>\inf _{M} u \lambda_{\Omega_{j}} \int_{\Omega_{j}} v_{j}+\inf _{\Omega_{j}}\left(f u^{N-1}\right) \int_{\Omega_{j}} v_{j}
$$

et donc par passage à la limite $\left|\lambda_{f}+\widetilde{R}\right| \sup _{M} u \geq \inf _{M} u \lambda_{f}>0 \quad\left(\lambda_{f}>0\right.$ puisque $f$ est négative en un point), on en déduit finalement $\lambda_{f} \neq-\widetilde{R}$. 


\section{DEMONSTRATION DU THEOREME 1}

Puisqu'il est équivalent de prouver l'existence d'une solution pour l'équation (1) avec la fonction $f$ ou avec $\alpha f, \alpha>0$, on considère la fonctionnelle:

$$
\left.F_{q}(u)=\int|\nabla u|^{2}+\widetilde{R} \int u^{2}-\int f u^{q} \text { où } q \in\right] 2, N[,
$$

$u$ est une fonction qui appartient à l'ensemble

$$
\mathscr{B}_{k, q}=\left\{u \in H_{1}^{2}, u \geq 0,\|u\|_{q}^{q}=k\right\}
$$

et on note $\mu_{k, q}=\inf _{u \in \mathscr{B}_{k, q}} F_{q}(u)$.

IV.1. $\mu_{k, q}$ est atteint. On considère une suite minimisante de fonctions de $\mathscr{B}_{k, q}$, soit $\left(u_{j}\right)_{j \in \mathbb{N}}$ telle que $\left\|u_{j}\right\|_{q}^{q}=k$, et $\lim _{j \rightarrow+\infty} F_{q}\left(u_{j}\right)=\mu_{k, q} \cdot u_{j}$ est une suite bornée dans $H_{1}^{2}$, car à partir d'un certain rang $F_{q}\left(u_{j}\right) \leq \mu_{k, q}+1$, et donc $\left\|\nabla u_{j}\right\|_{2}^{2} \leq \mu_{k, q}+1+k \sup f-k^{2 / q} \widetilde{R}$ et $\left\|u_{j}\right\|_{2}^{2} \leq k^{2 / q}$.

Par conséquent $u_{j}$ converge faiblement dans $H_{1}^{2}$, l'inclusion compacte de $H_{1}^{2}$ dans $L_{q}$ et l'unicité de la limite faible permettent d'affirmer qu'une soussuite toujours notée $u_{j}$ converge fortement dans $L_{q}$ vers une limite $u$. $u$ vérifie:

$$
\|u\|_{q}^{q}=k \text { et } \int \nabla u \nabla v+\widetilde{R} \int u v-\frac{q}{2} \int f u^{q-1} v=\lambda_{k, q} \int u^{q-1} v .
$$

Les théorèmes de régularité montrent qu'alors $u$ est continue. Evidemment, lorsque $k$ tend vers zéro, $\mu_{k, q}$ tend vers zéro. On peut préciser le comportement de $\mu_{k, q}$ en zéro grâce au lemme suivant:

Lemme 4. Lorsque $k$ est assez proche de zéro $\mu_{k, q}<0$.

En effet $\mu_{k, q} \leq F_{q}\left(k^{1 / q}\right) \leq \widetilde{R} k^{2 / q}-k \int f$. Donc, pour des normes $k$ assez petites $\mu_{k, q}$ est négatif. De plus la tangente à la courbe $k \rightarrow \mu_{k, q}$ est verticale en zéro.

Pour des normes $k$ grandes.

Si $f<0, F_{q}(u) \geq \widetilde{R} k^{2 / q}+k|\sup f|$, on en déduit immédiatement que $\mu_{k, q}$ est positif lorsque $k$ est assez grand.

Le cas le plus délicat est le cas sup $f=0$, on verra au cours de la démonstration de la proposition 2 qu'alors il existe $k_{0}$ tel que $\mu_{k, q}>0$ pour tout $k>k_{0}$.

Lemme 5. Si $\sup f>0, \mu_{k, q}$ est négatif pour $k$ assez grand. De plus $\mu_{k, q}$ est atteint pour des fonctions telles que $\int f u^{q}>0$.

En effet, si $\mu_{k, q}<\widetilde{R} k^{2 / q}<0$, une fonction $u$ qui réalise le minimum a la propriété $\int f u^{q}>0$. On considère alors une fonction $v$ à support inclus dans l'ouvert de la variété où $f>0$. On suppose $v \in C^{1}$ et $\|v\|_{q}^{q}=1$. Alors

$$
F_{q}\left(k^{1 / q} v\right)=\left(\|\nabla v\|_{2}^{2}+\widetilde{R}\|v\|_{2}^{2}\right) k^{2 / q}-k \int f v^{q} .
$$

Comme $\int f v^{q}>0, F_{q}\left(k^{1 / q} v\right)<\widetilde{R} k^{2 / q}$ pour $k$ assez grand. Donc $\mu_{k, q}$ est négatif à partir d'une certaine valeur de $k$. 
IV.2. Continuité de $\mu_{k, q}$.

Proposition 1. $\mu_{k, q}$ est une fonction continue de $k$.

Démonstration. Pour $k$ et $k^{\prime}$, soient $u$ et $u^{\prime}$ des fonctions de norme 1 dans $L_{q}$ telles que $F_{q}\left(k^{1 / q} u\right)=\mu_{k, q}$ et $F_{q}\left(k^{\prime 1 / q} u\right)=\mu_{k^{\prime}, q}$. Alors

$$
\begin{aligned}
\mu_{k^{\prime}, q}-\mu_{k, q}= & k^{2 / q}\left[\int\left|\nabla u^{\prime}\right|^{2}-|\nabla u|^{2}+\widetilde{R} \int u^{\prime 2}-u^{2}\right]-k \int f\left(u^{\prime q}-u^{q}\right) \\
& +\left(k^{\prime 2 / q}-k^{2 / q}\right)\left[\int\left|\nabla u^{\prime}\right|^{2}+\widetilde{R} \int u^{\prime 2}\right]-\left(k^{\prime}-k\right) \int f u^{\prime q} .
\end{aligned}
$$

Comme $k^{\prime}$ est pris dans un voisinage de $k, \int\left|\nabla u^{\prime}\right|^{2}+\widetilde{R} \int u^{\prime 2}$ et $\int f u^{\prime q}$ restent bornés. Par ailleurs, par définition de $\mu_{k, q}, F_{q}\left(k^{1 / q} u^{\prime}\right) \geq F_{q}\left(k^{1 / q} u\right)$.

On en déduit

$$
\liminf _{k^{\prime} \rightarrow k}\left(\mu_{k^{\prime}, q}-\mu_{k, q}\right) \geq 0 \text {. }
$$

Mais, en mettant $k^{\prime}$ en facteur:

$$
\begin{aligned}
\mu_{k^{\prime}, q}-\mu_{k, q}= & k^{\prime 2 / q}\left[\int\left|\nabla u^{\prime}\right|^{2}-|\nabla u|^{2}-\widetilde{R} \int u^{\prime 2}-u^{2}\right]-k^{\prime} \int f\left(u^{\prime q}-u^{q}\right) \\
& +\left(k^{\prime 2 / q}-k^{2 / q}\right)\left[\int|\nabla u|^{2}+\widetilde{R} \int u^{2}\right]-\left(k^{\prime}-k\right) \int f u^{q} .
\end{aligned}
$$

En utilisant cette fois la définition de $\mu_{k^{\prime}, q}$ on obtient

$$
\limsup _{k^{\prime} \rightarrow k}\left(\mu_{k^{\prime}, q}-\mu_{k, q}\right) \leq 0 \text {. }
$$

Par conséquent $\mu_{k, q}$ est une fonction continue de $k$.

IV.3. Etude de $\lambda_{f, \eta, q}$ dans le cas $e \int_{f \geq 0} 1>0$. On définit maintenant la quantité $\lambda_{f, \eta, q}=\inf _{u \in \mathscr{A}(\eta, q)}\|\nabla u\|_{2}^{2} /\|u\|_{2}^{2}$, avec

$$
\mathscr{A}(\eta, q)=\left\{u \in H_{1}^{2} / u \geq 0,\|u\|_{q}^{q}=1 \text {, et } \int\left|f^{-}\right| u^{q}=\eta \int\left|f^{-}\right|\right\},
$$

pour un réel $\eta>0$.

On suppose $\int_{f \geq 0} 1>0$ et on définit $\lambda_{f, \eta, q}^{\prime}=\inf _{u \in \mathscr{A}^{\prime}(\eta, q)}\|\nabla u\|_{2}^{2} /\|u\|_{2}^{2}$, avec

$$
\mathscr{A}^{\prime}(\eta, q)=\left\{u \in H_{1}^{2} / u \geq 0,\|u\|_{q}^{q}=1 \text {, et } \int\left|f^{-}\right| u^{q} \leq \eta \int\left|f^{-}\right|\right\} \text {. }
$$

$\lambda_{f, \eta, q}^{\prime}$ est une fonction décroissante de $\eta$, qui est majorée par $\lambda_{f}$ pour tout $\eta$, mais aussi par $\lambda_{f, \eta, q}$.

Soit alors une suite minimisante $v_{i}$ de fonctions de $\mathscr{A}^{\prime}(\eta, q)$ telle que $\left\|\nabla v_{i}\right\|_{2}^{2} /\left\|v_{i}\right\|_{2}^{2}$ tende vers $\lambda_{f, \eta, q}^{\prime}$. Les $v_{i}$ forment une suite bornée de $H_{1}^{2}$, cette suite converge faiblement dans $H_{1}^{2}$ donc fortement dans $L_{2}$ et $L_{q}$ (à une sous-suite près) vers $v$.

Donc $\|v\|_{q}^{q}=1, \int\left|f^{-}\right| v^{q} \leq \int\left|f^{-}\right| \eta$ et $\|v\|_{2}^{2}=\lim \left\|v_{i}\right\|_{2}^{2}$. Finalement, $v \in \mathscr{A}^{\prime}(\eta, q)$. Mais, comme $\|\nabla v\|_{2} \leq \lim \left\|\nabla v_{i}\right\|_{2},\|\nabla v\|_{2}^{2} /\|v\|_{2}^{2} \leq \lambda_{f, \eta, q}^{\prime}$ donc $\lambda_{f, \eta, q}^{\prime}$ est atteint.

De plus $\int\left|f^{-}\right| v^{q}=\int\left|f^{-}\right| \eta$. En effet si $\int\left|f^{-}\right| v^{q}<\int\left|f^{-}\right| \eta$, il existe une constante positive a telle que $\int\left|f^{-}\right|(v+a)^{q}=\int\left|f^{-}\right| \eta$ alors $\|v+a\|_{q}^{q} \geq 1$ et

$$
\frac{\|\nabla(v+a)\|_{2}^{2}}{\|v+a\|_{2}^{2}}<\frac{\|\nabla v\|_{2}^{2}}{\|v\|_{2}^{2}}=\lambda_{f, \eta, q}^{\prime} .
$$


Puisque $(v+a) /\|v+a\|_{2}^{2} \in \mathscr{A}^{\prime}(\eta, q)$, ceci est en contradiction avec la définition de $\lambda_{f, \eta, q}^{\prime}$.

On a donc montré que $\lambda_{f, \eta, q}^{\prime}$ et $\lambda_{f, \eta, q}$ sont égaux, donc $\lambda_{f, \eta, q}$ est une fonction décroissante de $\eta$, qui est majorée par $\lambda_{f}$ pour tout $\eta$, et $\lambda_{f, \eta, q}$ est atteint par une fonction de $\mathscr{A}(\eta, q)$, avec $\mathscr{A}(\eta, q)=\left\{u \in H_{1}^{2} / u \geq 0\right.$, $\|u\|_{q}^{q}=1$, et $\left.\int\left|f^{-}\right| u^{q}=\eta \int\left|f^{-}\right|\right\}$.

Pour étudier la convergence de $\lambda_{f, \eta, q}$ lorsque $\eta$ décroît, on démontre deux lemmes:

Lemme 6. Soit $q \in] 2, N\left[\right.$ lorsque $\eta$ tend vers zéro $\lambda_{f, \eta, q}$ tend vers $\lambda_{f}$.

Démonstration. Puisque $\lambda_{f, \eta, q}$ est atteint par une fonction que l'on notera $v_{\eta, q}$. Lorsque $\eta$ varie, les $v_{\eta, q}$ forment une suite bornée de $H_{1}^{2}$. En effet, $M$ est de volume 1, et $\left\|v_{\eta, q}\right\|_{q}^{q}=1$. Donc $\left\|v_{\eta, q}\right\|_{2}^{2} \leq 1$ et $\left\|\nabla v_{\eta, q}\right\|_{2}^{2} \leq \lambda_{f, \eta, q} \leq \lambda_{f}$. Donc, lorsque $\eta$ tend vers zéro, $v_{\eta, q}$ converge à une sous-suite près faiblement dans $H_{1}^{2}$ vers $v_{q}$, fortement dans $L_{2}$ et $L_{q}$. Par conséquent $\left\|v_{q}\right\|_{q}^{q}=1$, $\int\left|f^{-}\right| v^{q}=0, v_{q} \geq 0$, donc $v_{q} \in \mathscr{A}(\eta, q)$ et $\left\|v_{q}\right\|_{2}^{2}=\lim \left\|v_{\eta, q}\right\|_{2}^{2}$ d'autre part:

$$
\begin{aligned}
\left\|\nabla v_{q}\right\|_{2}^{2} & \leq \lim \left(\lambda_{f, \eta, q}\left\|v_{\eta, q}\right\|_{2}^{2}\right) \\
& \leq \lambda_{f} \lim \left\|v_{\eta, q}\right\|_{2}^{2} \\
& \leq \lambda_{f}\left\|v_{q}\right\|_{2}^{2} .
\end{aligned}
$$

Donc $\left\|\nabla v_{q}\right\|_{2}^{2} /\left\|v_{q}\right\|_{2}^{2}=\lambda_{f}$ par définition de $\lambda_{f}$. Ce qui montre que $\lambda_{f, \eta, q}$ tend vers $\lambda_{f}$ lorsque $q$ est fixé.

Lemme 7. Soit $\varepsilon>0$, il existe $\eta_{0}$ tel que pour tout $\eta<\eta_{0}$, il existe $q_{\eta}$ tel que pour tout $q>q_{\eta}, \lambda_{f, \eta, q} \geq \lambda_{f}-\varepsilon$.

Démonstration. On va raisonner par l'absurde. Supposons qu'il existe un $\varepsilon>0$ tel que, pour tout $\eta_{0}$, il existe $\eta<\eta_{0}$ et pour tout $q_{\eta}$, il existe une valeur de $q>q_{\eta}$ telle que $\lambda_{f, \eta, q}<\lambda_{f}-\varepsilon$.

Soit toujours $v_{\eta, q}$ une fonction qui réalise $\lambda_{f, \eta, q}$, on a $\left\|v_{\eta, q}\right\|_{q}^{q}=1$ donc $\left\|v_{\eta, q}\right\|_{2}^{2} \leq 1$ et $\left\|\nabla v_{\eta, q}\right\|_{2}^{2} /\left\|v_{\eta, q}\right\|_{2}^{2}=\lambda_{f, \eta, q}$. Ainsi $\eta$ étant convenablement choisi, il existe une suite de valeurs de $q$ qui tend vers $N$ telle que pour chaque $q$ de cette suite la fonction $v_{\eta, q}$ associée vérifie

$$
\frac{\left\|\nabla v_{\eta, q}\right\|_{2}^{2}}{\left\|v_{\eta, q}\right\|_{2}^{2}}=\lambda_{f, \eta, q}<\lambda_{f}-\varepsilon .
$$

Ces $v_{\eta, q}$ forment une suite bornée de $H_{1}^{2}$ qui converge faiblement dans $H_{1}^{2}$, fortement dans $L_{2}$ vers $v_{\eta}$. On a donc $\left\|\nabla v_{\eta}\right\|_{2}^{2} \leq \liminf \left\|\nabla v_{\eta}, q\right\|_{2}^{2}$. La convergence forte dans $L_{2}$ permet d'affirmer $\left\|\nabla v_{\eta}\right\|_{2}^{2} \leq\left(\lambda_{f}-\varepsilon\right) \int v_{\eta}^{2}$, mais l'inégalité de Sobolev $1 \leq\left(K_{1} \lambda_{f}+A_{1}\right) \int v_{\eta, q}^{2}$ implique

$$
\int v_{\eta}^{2} \geq \frac{1}{K_{1} \lambda_{f}+A_{1}}
$$

On vérifie alors aisément que $\int v_{\eta}^{N} \leq 1$ et $\int\left|f^{-}\right| v_{\eta}^{2} \leq \eta \int\left|f^{-}\right|$. En effet, pour $k<N$ fixé, $\forall q \geq k$

$$
\begin{aligned}
\int v_{\eta, q}^{k} \leq 1 \text { et } \int\left|f^{-}\right| v_{\eta, q}^{k} & \leq\left[\int\left|f^{-}\right| v_{\eta, q}^{q}\right]^{k / q}\left[\int\left|f^{-}\right|\right]^{1-k / q} \\
& \leq \eta^{k / q} \int\left|f^{-}\right| .
\end{aligned}
$$


$k$ étant toujours fixé on peut faire tendre $q$ vers $N$

$$
\int v_{\eta}^{k} \leq 1 \text { et } \int\left|f^{-}\right| v_{\eta}^{k} \leq \eta^{k / N} \int\left|f^{-}\right| \text {. }
$$

Sur $A=\left\{x \in M / v_{\eta}(x)>1\right\}$, le lemme de Fatou appliqué à $v_{\eta}^{k}$ et $\left|f^{-}\right| v_{\eta}^{k}$ impose $\lim _{k \rightarrow N} \int_{A} v_{\eta}^{k}=\int v_{\eta}^{N}$ et $\lim _{k \rightarrow N} \int_{A}\left|f^{-}\right| v_{\eta}^{k}=\int_{A}\left|f^{-}\right| v_{\eta}^{N}$. Sur $M \backslash A$ la convergence dominée permet de conclure. Faisons maintenant varier $\eta$, considérons une suite de valeurs de $\eta$ qui tend vers zéro, telle que pour chaque $\eta$ de cette suite, pour tout $q_{\eta}$, il existe une valeur de $q>q_{\eta}$ telle que $\lambda_{f, \eta, q}<\lambda_{f}-\varepsilon$. On peut, d'après ce qui précède, associer à chacune de ces valeurs de $\eta$ une fonction $v_{\eta}$. La suite des fonctions $v_{\eta}$ est bornée dans $H_{1}^{2}$ et

$$
\int v_{\eta}^{2} \geq \frac{1}{K_{1} \lambda_{f}+A_{1}}
$$

donc lorsque $\eta$ tend vers zéro, $v_{\eta}$ converge faiblement dans $H_{1}^{2}$ et fortement dans $L_{2}$ vers $v$ positive et non identiquement nulle, qui vérifie

$$
\int|\nabla v|^{2} \leq\left(\lambda_{f}-\varepsilon\right)\|v\|_{2}^{2}
$$

Mais $0 \leq \int\left|f^{-}\right| v^{N} \leq \lim _{\eta \rightarrow 0} \int\left|f^{-}\right| v_{\eta}^{N} \leq \eta \int\left|f^{-}\right|$implique $\int\left|f^{-}\right| v=0$. Par conséquent $v \in \mathscr{A}$ et par définition de $\lambda_{f}, \int|\nabla v|^{2} /\|v\|_{2}^{2} \geq \lambda_{f}$, ce qui est contradictoire. Autrement dit $\forall \varepsilon>0, \exists \eta_{0}$ tel que $\forall \eta<\eta_{0}, \int\left|\nabla v_{\eta}\right|^{2} /\left\|v_{\eta}\right\|_{2}^{2} \geq$ $\lambda_{f}-\varepsilon$.

$\exists \eta_{0}$ tel que $\forall \eta<\eta_{0}$ il existe $q_{\eta}$ tel que $\forall q>q_{\eta}, \lambda_{f, \eta, q} \geq \lambda_{f}-\varepsilon$.

Si maintenant $\sup f=0$ et $\int_{f \geq 0} 1=0, \lambda_{f}$ n'est plus défini. On va montrer que l'on obtient l'équivalent des lemmes 6 et 7 en remplaçant $\lambda_{f}$ par $+\infty$.

IV.4. Etude de $\lambda_{f, \eta, q}$ dans le cas $\int_{f \geq 0} 1=0$, sup $f=0$. Si $\int_{f \geq 0} 1=0$ et donc $\sup f=0$, on peut toujours définir $\lambda_{f, \eta, q}$ pour $\eta \neq 0$. $\bar{\lambda}_{f, \eta, q}=$ $\inf _{u \in \mathscr{A}(\eta, q)}\|\nabla u\|_{2}^{2} /\|u\|_{2}^{2}$, avec $\mathscr{A}(\eta, q)=\left\{u \in H_{1}^{2} / u \geq 0,\|u\|_{q}^{q}=1\right.$, et $\left.\int\left|f^{-}\right| u^{q}=\eta \int\left|f^{-}\right|\right\}$. A $\eta$ fixé, pour $\left.q \in\right] 2, N\left[, \lambda_{f, \eta, q}\right.$ existe, en effet $\mathscr{A}(\eta, q)$ est non vide puisqu'il existe toujours une fonction $u C^{\infty}$ telle que $\|u\|_{q}^{q}=1$ dont le support soit inclus dans l'ouvert $\left\{x \in M,\left|f^{-}\right|(x)<\eta \int\left|f^{-}\right|\right\}$. La même démonstration que dans le cas $\int_{f \geq 0} 1>0$, prouve que $\lambda_{f, \eta, q}$ est atteint par une fonction de $\mathscr{A}(\eta, q)$, et que $\lambda_{f, \eta, q}$ décroît lorsque $\eta$ croît.

On a alors le lemme 8

Lemme 8. Soit $q \in] 2, N\left[\right.$ lorsque $\eta$ tend vers zéro $\lambda_{f, \eta, q}$ tend vers l'infini.

Démonstration. $\lambda_{f, \eta, q}$ est atteint par une fonction que l'on notera $v_{\eta, q}$. Supposons que lorsque $\eta$ tend vers zéro, on puisse trouver une suite de $v_{\eta, q}$ qui forment une suite bornée de $H_{1}^{2}$.

Lorsque $\eta$ tend vers zéro, les $v_{\eta, q}$ convergent, à une sous-suite près, faiblement dans $H_{1}^{2}$ vers $v_{q}$, fortement dans $L_{2}$ et $L_{q}$.

Par conséquent, $\left\|v_{q}\right\|_{q}^{q}=1$ et $\int\left|f^{-}\right| v_{q}^{q}=0$. Comme cette dernière égalité entraine $v_{q}=0$ presque partout, elle est contradictoire avec la première. Donc $\lambda_{f, \eta, q}$ tend vers l'infini lorsque $\eta$ tend vers zéro, à $q$ fixé.

On peut aussi montrer l'équivalent du lemme 7 
Lemme 9. Il existe $\eta_{0}$ tel que pour tout $\eta<\eta_{0}$, il existe $q_{\eta}$ tel que pour tout $q>q_{\eta} \lambda_{f, \eta, q}>|\widetilde{R}|$.

Démonstration. On raisonne de nouveau par l'absurde.

Supposons qu'il existe une suite de valeurs de $\eta,\left(\eta_{j}\right)_{j \in \mathbb{N}}$ qui tende vers zéro, telle qu'il existe $\left.q_{j} \in\right] 2, N$ [ tel que: $\lambda_{f, \eta_{j}, q_{j}} \leq|\widetilde{R}| . \quad \lambda_{f, \eta_{j}, q_{j}}$ est atteint par une fonction $v_{j}$ qui vérifie $\left\|v_{j}\right\|_{q_{j}}^{q_{j}}=1$ et $\left\|\nabla v_{j}\right\|_{2}^{2} \leq \lambda_{f, \eta_{j}, q_{j}}$. La suite $v_{j}$ est donc bornée dans $H_{1}^{2}$, elle converge (à une sous suite près) faiblement dans $H_{1}^{2}$, fortement dans $L_{2}$ vers une fonction $v$.

De plus, toujours à une sous-suite près, quelque soit la limite $q$ des $q_{j}, q \in$ $[2, N]$, puisque la suite converge faiblement dans $L_{q}$, on a:

$$
0 \leq \int\left|f^{-}\right| v^{q} \leq \lim _{j \rightarrow \infty} \int\left|f^{-}\right| v_{j}^{q_{j}} \leq \eta_{j} \int\left|f^{-}\right| .
$$

Donc $\int\left|f^{-}\right| v^{q}=0, v$ est presque partout nulle, $\|v\|_{2}=0$. Ainsi $\lim \left\|v_{j}\right\|_{2}^{2}=0$ et l'inégalité de Sobolev

$$
K_{1}\left\|\nabla v_{j}\right\|_{2}^{2} \geq 1-A_{1}\left\|v_{j}\right\|_{2}^{2}
$$

contredit le fait que $\lambda_{f, \eta_{j}, q_{j}}$ reste borné.

Ces lemmes établis on peut démontrer la proposition 2.

IV.5. $\mu_{k, q}$ est positif pour une certaine valeur de $k$.

Proposition 2. Soit $q \in] 2, N\left[\right.$, on rappelle, $\widetilde{R}=\frac{n-2}{4(n-1)} R$.

(a) Supposons $\sup f>0$ et $\lambda_{f}>|\widetilde{R}|$, il existe $\eta$, tel que $\varepsilon=\lambda_{f, \eta, q}+\widetilde{R}>0$. On pose

$$
C_{q}=\frac{\eta}{8} \inf \left(\frac{\varepsilon}{|\widetilde{R}|\left[A_{1}+K_{1}(|\widetilde{R}|+\varepsilon)\right]}, 1\right)
$$

où $A_{1}$ et $K_{1}$ sont les constantes de l'inégalité de Sobolev. Si sup $f / \int\left|f^{-}\right|<C_{q}$, où $C_{q}$ ne dépend que de $f^{-} / \int\left|f^{-}\right|$, alors il existe un intervalle $I_{q}=\left[k_{1, q}, k_{2, q}\right]$ tel que $\forall k \in I_{q}, \mu_{k, q} \geq 0$.

(b) Si $\sup f=0$, soit $\int_{f \geq 0} 1=0$, soit si $\int_{f \geq 0} 1 \neq 0$, on suppose $\lambda_{f}>|\widetilde{R}|$, alors il existe un intervalle $I_{q}=\left[k_{1, q},+\infty\left[\right.\right.$ tel que $\forall k \in I_{q}, \mu_{k, q} \geq 0$.

Démonstration. Si sup $f>0$, dans les conditions de la proposition, d'après les lemmes 6 et 8 , il existe $\eta$, tel que $\varepsilon=\lambda_{f, \eta, q}+\widetilde{R}>0$.

On suppose que $f$ vérifie les conditions de la proposition. Soit alors $u$, une fonction de $H_{1}^{2}$, positive ou nulle sur $M$, telle que: $\|u\|_{q}^{q}=k$ avec $k^{(q-2) / q}>$ $2|\widetilde{R}| / \eta \int\left|f^{-}\right|$. On pose $k_{1, q}=\left[2|\widetilde{R}| / \eta \int\left|f^{-}\right|\right]^{q /(q-2)}$.

Deux cas se présentent:

Soit $\int\left|f^{-}\right| u^{q} \geq \eta k \int\left|f^{-}\right|$. Mais alors, on peut minorer

$$
G_{q}(u)=\|\nabla u\|_{2}^{2}+\widetilde{R}\|u\|_{2}^{2}+\int\left|f^{-}\right| u^{q} .
$$


En effet:

$$
\begin{aligned}
G_{q}(u) & \geq \widetilde{R}\|u\|_{q}^{2}+\int\left|f^{-}\right| \eta k \\
& \geq|\widetilde{R}| k^{2 / q}\left[\frac{\eta \int\left|f^{-}\right|}{|\widetilde{R}|} k^{1-2 / q}-1\right] \\
& \geq|\widetilde{R}| k^{2 / q} .
\end{aligned}
$$

Soit $\int\left|f^{-}\right| u^{q}<\eta k \int\left|f^{-}\right|$mais alors: $\|\nabla u\|_{2}^{2} /\|u\|_{2}^{2}>\lambda_{f, \eta, q}$, et on peut minorer $G_{q}(u)$ ainsi:

$$
\begin{aligned}
G_{q}(u) & \geq\left(\lambda_{f, \eta, q}+\tilde{R}\right)\|u\|_{2}^{2}+\int\left|f^{-}\right| u^{q} \\
& \geq \varepsilon\|u\|_{2}^{2}+\int\left|f^{-}\right| u^{q} .
\end{aligned}
$$

On pose alors $\alpha+\beta=\varepsilon, \alpha=\beta A_{1} /|\widetilde{R}| K_{1}$, comme

$$
\|u\|_{2}^{2}=\frac{1}{|\widetilde{R}|}\left[\|\nabla u\|_{2}^{2}+\int\left|f^{-}\right| u^{q}-G_{q}(u)\right]
$$

d'après la définition de $\alpha$ et $\beta$, on a:

$$
G_{q}(u) \geq \alpha\|u\|_{2}^{2}+\frac{\beta}{|\widetilde{R}|}\left[\|\nabla u\|_{2}^{2}+\int\left|f^{-}\right| u^{q}-G_{q}(u)\right]+\int\left|f^{-}\right| u^{q}
$$

et donc

$$
\begin{aligned}
\left(1+\frac{\beta}{|\widetilde{R}|}\right) G_{q}(u) & \geq \alpha\|u\|_{2}^{2}+\frac{\beta}{|\widetilde{R}|}\|\nabla u\|_{2}^{2} \\
& \geq \frac{\beta}{|\widetilde{R}|}\left[\|\nabla u\|_{2}^{2}+\frac{\alpha|\widetilde{R}|}{\beta}\|u\|_{2}^{2}\right]
\end{aligned}
$$

par construction $\alpha|\widetilde{R}| / \beta=A_{1} / K_{1}$ d'où comme $K_{1}\|\nabla u\|_{2}^{2}+A_{1}\|u\|_{2}^{2} \geq\|u\|_{q}^{2}=$ $k^{2 / q}$.

$$
G_{q}(u) \geq \frac{\beta}{|\widetilde{R}|} \frac{k^{2 / q}}{K_{1}}\left(1+\frac{\beta}{|\widetilde{R}|}\right)^{-1}
$$

et finalement puisque

$$
\beta=\frac{K_{1}|\widetilde{R}| \varepsilon}{A_{1}+K_{1}|\widetilde{R}|}, \quad G_{q}(u) \geq \frac{\varepsilon}{A_{1}+K_{1}(|\widetilde{R}|+\varepsilon)} k^{2 / q}
$$

si $\sup f=0$, on a $\forall k>k_{1, q}, \mu_{k, q}>0$.

Si $\sup f>0$, posons

$$
m=\inf \left(\frac{\varepsilon}{A_{1}+K_{1}(|\widetilde{R}|+\varepsilon)},|\widetilde{R}|\right) .
$$

$F_{q}(u)=G_{q}(u)-\int f^{+} u^{q} \geq G_{q}(u)-\sup f k$ et $\forall k>k_{1, q}, F_{q}(u) \geq m k^{2 / q}-$ $k \sup f$. On a

$$
F_{q}(u)>\frac{1}{2} m k^{2 / q}>0, \quad \text { si } k<\left[\frac{m}{2 \sup f}\right]^{q /(q-2)} .
$$


Mais puisque l'on a posé $\sup f \leq \int\left|f^{-}\right| C_{q}$, avec $C_{q}=m \eta / 8|\widetilde{R}|$, l'inégalité est vérifiée si

$$
k<\left[\frac{m}{2 \int|f-| C_{q}}\right]^{q /(q-2)}=\left[\frac{4|\widetilde{R}|}{\eta \int|f-|}\right]^{q /(q-2)}=2^{q /(q-2)} k_{1, q},
$$

avec toujours $k_{1, q}=\left[2|\tilde{R}| / \eta \int\left|f^{-}\right|\right]^{q /(q-2)}$. On posera $k_{2, q}=2^{n / 2} k_{1, q}$. Ce qui démontre la proposition.

IV.6. Fin de la démonstration du théorème 1.

Remarque. Lorsque l'on considère une suite de valeurs de $q$ qui tend vers $N$, la suite des valeurs $C_{q}$ ne tend pas vers 0 ; de même si sup $f=0, k_{1, q}$ ne tend pas vers l'infini et si sup $f>0, k_{1, q}$ ne tend pas vers $k_{2, q}$, et le sup des $\mu_{k, q}$ ne tend pas vers zéro.

En effet, si on fait tendre $q$ vers $N$, d'après le lemme 7, on peut choisir une valeur de $\eta$, telle que il existe $q_{\eta}$ qui réalise pour tout $q>q_{\eta}, \lambda_{f, \eta, q}+\widetilde{R} \geq$ $\varepsilon>0$. Alors, d'après la proposition 2 , si

$$
\sup f<C=\frac{\eta}{8} \inf \left(\frac{\varepsilon}{|\widetilde{R}|\left[A_{1}+K_{1}(|\widetilde{R}|+\varepsilon)\right]}, 1\right)
$$

il existe une suite d'intervalles $I_{q}$, dont la longueur ne tend pas vers zéro, tels que pour tout $q, \forall k \in I_{q}, \mu_{k, q} \geq 0$.

Pour tout $q \in] q_{\eta}, N$ [, que $f$ prenne des valeurs positives ou non, on a donc montré que sous les hypothèses du théorème, il existe toujours un intervalle $\left[l_{q}, l_{q}^{\prime}\right]$ tel que $\forall k \in\left[l_{q}, l_{q}^{\prime}\right], \mu_{k, q}>0 . l_{q}$ et $l_{q}^{\prime}$ dépendent de $f$ et de $q$, mais pour une fonction $f$ donnée lorsque $q$ tend vers $N, l_{q}^{\prime}-l_{q}$ ne tend pas vers zéro, en effet on peut choisir $l_{q}$ tel que $l_{q} \rightarrow l=\left[2|\widetilde{R}| / \eta \int\left|f^{-}\right|\right]^{n / 2}$ et $l_{q}^{\prime} \rightarrow 2^{n / 2} l$. De plus, d'après la démonstration précédente, si sup $f>0$, en posant $\mu_{k_{0}, q}=\sup _{k \in \mathbb{R}^{+}} \mu_{k, q}$, il est clair que lorsque $q$ tend vers $N, \mu_{k_{0}, q}$ ne tend pas vers zéro. On pose alors

$$
\mu_{k_{q}, q}=\inf _{u \in \mathscr{D}_{k, q}} F_{q}(u)
$$

avec

$$
\mathscr{D}_{k, q}=\left\{u \in H_{1}^{2}, u \geq 0,\|u\|_{q}^{q} \leq l_{q}^{\prime}\right\}
$$

$\mu_{k_{q}, q}$ est atteint pour une fonction $u_{q}$ qui satisfait $\left\|u_{q}\right\|_{q}^{q}=k_{q}$ et $F_{q}\left(u_{q}\right)=$ $\mu_{k_{q}, q} \cdot \mu_{k, q}$ étant négatif lorsque $k$ est proche de zéro, $\mu_{k_{q}, q}<0$. On en déduit que $k_{q}$ est inférieur à $l_{q}$, donc à $l_{q}^{\prime}$, puisque $\forall k \in\left[l_{q}, l_{q}^{\prime}\right], \mu_{k, q}>0$.

$u_{q}$ est donc un point critique de la fonctionnelle et les théorèmes de régularité montrent qu'alors $u_{q}$ est une solution $C^{\infty}$ de l'équation (2). Comme il apparaît clairement dans la démonstration de la proposition 2 , les $u_{q}$ sont majorés dans $L_{q}$.

En effet puisque $k_{q}<k_{1, q}=\left[2|\widetilde{R}| / \eta \int\left|f^{-}\right|\right]^{q /(q-2)}$ on a $k_{q}<C^{\prime}$ avec

$$
C^{\prime}=\sup \left[1,\left[\frac{2|\widetilde{R}|}{\eta \int\left|f^{-}\right|}\right]^{q / n}\right] \text {. }
$$


Les $u_{q}$ sont des fonctions strictement positives (Aubin [3, Proposition 3.75]). Ces fonctions vérifient:

$$
\Delta u_{q}+\widetilde{R} u_{q}=\frac{q}{2} f u_{q}^{q-1}
$$

Ainsi lorsque $u_{q}$ est minimale $\Delta u_{q} \leq 0$ et par conséquent

(a) lorsque en un point $P$ de $M u_{q}$ est minimale $f(P)<0$.

(b) $f(P) u_{q}^{q-1}(P) \leq \frac{2}{q} \widetilde{R} u_{q}(P)$ donc

$$
u_{q}^{q-2}(P) \geq \frac{2}{q} \frac{\tilde{R}}{f(P)} \geq \frac{2}{N} \frac{\tilde{R}}{\inf f}>0 .
$$

Ainsi les $u_{q}$ sont uniformément minorées par $m^{\prime}>0$. Il reste à montrer que les $\mu_{k_{q}, q}$ sont uniformément bornés pour $q \in\left[q_{\eta}, N\right]$. D'une part $\mu_{k_{q}, q}<0$, d'autre part

$$
\begin{aligned}
\mu_{k_{q}, q} & \geq \widetilde{R} k_{q}^{2 / q}-\sup f k_{q} \geq \widetilde{R} C^{\prime 2 / q}-\sup f C^{\prime} \\
& \geq(\widetilde{R}-\sup f) C^{\prime}, \quad \operatorname{car} \frac{2}{q}<1 .
\end{aligned}
$$

On en déduit que la suite $u_{q}$ est bornée dans $H_{1}^{2}$. En effet

$$
\begin{aligned}
\left\|u_{q}\right\|_{2}^{2} \leq C^{\prime 2 / q}, \quad \text { et }\left\|\nabla u_{q}\right\|_{2}^{2} & \leq \mu_{k_{q}, q}+\int f u_{q}^{q}-\widetilde{R}\left\|u_{q}\right\|_{2}^{2} \\
& \leq \sup f C^{\prime} .
\end{aligned}
$$

A une sous-suite près, $u_{q}$ converge alors faiblement dans $H_{1}^{2}$ vers une fonction $u$, l'inclusion compacte de $H_{1}^{2}$ dans $L_{2}$ et l'unicité de la limite faible impliquent alors la convergence vers $u$ des $u_{q}$ dans $L_{2}$ et presque partout.

Par ailleurs pour tout $q \in\left[q_{0}, N\right]$ :

$$
\int \nabla u_{q} \nabla v+\widetilde{R} \int u_{q} v-\frac{q}{2} \int f u_{q}^{q-1} v=0, \quad \forall v \in H_{1}^{2}
$$

La convergence faible dans $H_{1}^{2}$ et forte dans $L_{N-1}$ impliquent

$$
\int \nabla u \nabla v+\widetilde{R} \int u v-\frac{N}{2} \int f u^{N-1} v=0, \quad \forall v \in H_{1}^{2} .
$$

Donc $u$ satisfait faiblement l'équation

$$
\Delta u+\widetilde{R} u=\frac{N}{2} f u^{N-1}
$$

Les théorèmes de régularité assurent qu'alors $u$ est $C^{\infty}$ et la convergence presque partout des $u_{q}$ vers $u$ entraîne $u(x) \geq m^{\prime}$ pour tout $x \in M, u$ est une solution non triviale de l'équation (1).

\section{DEMONSTRATION DU THÉOREME 2}

On suppose ici $\sup f>0$, et $\left(\sup f^{+}\right) / \int\left|f^{-}\right|<C$. Dans les conditions du théorème on a vu qu'il existe un intervalle $I_{q}$, tel que si $k \in I_{q}, \mu_{k, q}$ soit positif. Par ailleurs, pour $k$ proche de zéro $\mu_{k, q}$ est négatif, de même que lorsque $k$ tend vers l'infini.

On en déduit que $\mu_{k, q}$ possède un minimum et un maximum. Le minimum (relatif) est atteint, on l'a montré, par une fonction solution de l'équation (1). Pour montrer que l'on obtient une autre solution, on utilise le lemme du col. 
Lemme 10. La condition de Palais-Smale est vérifiée.

On raisonne par l'absurde. Supposons qu'il existe une suite de fonctions $v_{n}$ telle que $F\left(v_{n}\right)$ tend vers une limite finie $C, F\left(v_{n}\right)$ tend vers zéro et $v_{n}$ tend vers l'infini en norme $H_{1}^{2}$.

On a donc pour tout $\xi$ dans $H_{1}^{2}$ :

$$
\left\{\begin{array}{l}
\int \nabla v_{n} \nabla \xi+\widetilde{R} \int v_{n} \xi-\frac{q}{2} \int f v_{n}^{q-1} \xi=\varepsilon\|\xi\|, \\
\int\left|\nabla v_{n}\right|^{2}+\widetilde{R} \int v_{n}^{2}-\int f v_{n}^{q} \rightarrow C,
\end{array}\right.
$$

où \|\| désigne la norme dans $H_{1}^{2}$. Donc en particulier pour $\xi=v_{n}$.

$$
\left\{\begin{array}{l}
\int\left|\nabla v_{n}\right|^{2}+\widetilde{R} \int v_{n}^{2}-\frac{q}{2} \int f v_{n}^{q}=\varepsilon\left\|v_{n}\right\|, \\
\int\left|\nabla v_{n}\right|^{2}+\widetilde{R} \int v_{n}^{2}-\int f v_{n}^{q} \rightarrow C .
\end{array}\right.
$$

On en déduit $\forall \varepsilon>0, \exists N_{0} / \forall n>N_{0}$

$$
\left\{\begin{array}{l}
\left|\left(\frac{q}{2}-1\right) \int f v_{n}^{q}-C\right| \leq \varepsilon+\varepsilon\left\|v_{n}\right\|, \\
\left|\left(\frac{q}{2}-1\right)\left[\int\left|\nabla v_{n}\right|^{2}+\widetilde{R} \int v_{n}^{2}\right]-\frac{q}{2} C\right| \leq \frac{q}{2} \varepsilon+\varepsilon\left\|v_{n}\right\| .
\end{array}\right.
$$

Soit alors $k$ une norme $L_{q}$ telle que $\mu_{k, q}$ soit positif. On pose $w_{n}=k^{1 / q} \frac{v_{n}}{\left\|v_{n}\right\|_{q}}$, on a alors

$$
\left\{\begin{array}{l}
\left|\left(\frac{q}{2}-1\right) \int f w_{n}^{q}-\frac{k C}{\left\|v_{n}\right\|_{q}^{q}}\right| \leq \frac{k \varepsilon}{\left\|v_{n}\right\|_{q}^{q}}+\varepsilon \frac{\left\|w_{n}\right\|_{n}}{\left\|v_{n}\right\|_{q}^{q-1}}, \\
\left|\left(\frac{q}{2}-1\right)\left[\int\left|\nabla w_{n}\right|^{2}+\widetilde{R} \int w_{n}^{2}\right]-\frac{q}{2} \frac{k^{2 / q} C}{\left\|v_{n}\right\|_{q}^{2}}\right| \leq \frac{q}{2} \frac{k^{2 / q}}{\left\|v_{n}\right\|_{q}^{2}} \mid+k^{1 / q} \varepsilon\left\|w_{n}\right\|
\end{array}\right.
$$

Puisque $\left\|w_{n}\right\|_{2}^{2}$ est bornée, la deuxième équation entraine que $w_{n}$ est une suite bornée de $H_{1}^{2}$. Mais alors, si $\left\|v_{n}\right\|_{q}$ tend vers l'infini, les deux équations cidessus entraînent $F\left(w_{n}\right) \rightarrow 0$. Par ailleurs, comme $\left\|w_{n}\right\|_{q}^{q}=k$, on a $F\left(w_{n}\right) \geq$ $\mu_{k, q}$, et donc $\mu_{k, q} \leq 0$ mais on a choisi $k$ de telle sorte que $\mu_{k, q}>0$, on obtient donc une contradiction. Une telle suite est donc bornée dans $H_{1}^{2}$. Puisque $q<N$, les inclusions de Sobolev sont compactes, on en déduit que la condition de Palais-Smale est vérifiée.

Soit $k_{0}$ la norme telle que $\mu_{k_{0}, q}$ soit maximum. Soient $k_{1}$ et $k_{2}$ des normes telles que

$$
\begin{array}{ll}
\mu_{k_{1}, q}=0 ; & k_{1}<k_{0}, \\
\mu_{k_{2}, q}=0 ; & k_{2}>k_{0} .
\end{array}
$$

Soient

$$
\Gamma \in\left\{h \in C\left([0,1], H_{1}^{2}\right) / h(0)=u_{k_{1}, q}, h(1)=u_{k_{2}, q}\right\}
$$

et

$$
\mu_{q}=\inf _{h \in \Gamma} \max _{y \in[0,1]} F_{q}(h(y)) .
$$

Si $\mu_{q}$ n'était pas un niveau critique, il existerait $\varepsilon$, inférieur à $\mu_{q} / 2$, tel que $F_{q}^{-1}\left\{\left[\mu_{q}-\varepsilon, \mu_{q}+\varepsilon\right]\right\}$ ne contienne pas de point critique.

Mais alors, en reprenant la démonstration d'Ambrosetti-Rabinowitz [1], il existerait $\eta_{t}, t \in[0,1]$, continue en $t$, de $H_{1}^{2}$ dans $H_{1}^{2}$, vérifiant:

(1) $\eta_{0}(u)=u, \forall u \in H_{1}^{2}$.

(2) $\eta_{t}(u)=u, \forall u \in H_{1}^{2}, u \notin F_{q}^{-1}\left\{\left[\mu_{q}-\varepsilon, \mu_{q}+\varepsilon\right]\right\}$. 
(3) $\eta_{t}$ est un homéomorphisme de $H_{1}^{2}$.

(4) $F_{q}\left(\eta_{t}(u)\right) \leq F_{q}(u), \forall u \in H_{1}^{2}$.

(5) $\eta_{1}\left(\left\{u / F_{q}(u) \leq \mu+\varepsilon\right\}\right)$ est inclus dans $\left\{u / F_{q}(u) \leq \mu_{q}-\varepsilon\right\}$.

Soit alors un chemin $h \in \Gamma$ tel que $\max _{y \in[0,1]} F_{q}(h(y))<\mu_{q}+\varepsilon$. Comme $F_{q}\left(u_{k_{1}, q}\right) \leq \mu_{q}-\varepsilon$ et $F_{q}\left(u_{k_{2}, q}\right) \leq \mu_{q}-\varepsilon$,

$$
\eta_{1}\left(u_{k_{1}, q}\right)=u_{k_{1}, q} \text { et } \eta_{1}\left(u_{k_{2}, q}\right)=u_{k_{2}, q} \text {. }
$$

Donc $\eta_{1}(h) \in \Gamma$ mais $\max _{y \in[0,1]} F_{q}\left(\eta_{1}(h(y)) \leq \mu_{q}-\varepsilon\right.$ ce qui serait une contradiction.

On en déduit que $\mu_{q}$ est un niveau critique de la fonctionnelle, comme par ailleurs on a $\mu_{q} \geq \mu_{k_{0}, q}>0$, il s'ensuit que l'équation (2) admet deux solutions distinctes non nulles.

\section{BIBLIOGRAPHIE}

1. A. Ambrosetti et P. Rabinowitz, Dual variational methods in critical point theory and applications, J. Funct. Anal. 14 (1973), 349-381.

2. Th. Aubin, Equations différentielles non linéaires et problème de Yamabe concernant la courbure scalaire, J. Math. Pures Appl 55 (1976), 269-296.

3. __ Nonlinear analysis on manifolds-Monge-Ampere equations, Grundlehren der Math. Wissenschaften, vol. 252, Springer-Verlag, Berlin, 1982.

4. J. L. Kazdan et F. W. Warner, Scalar curvature and conformal deformation of Riemannian structure, J. Differential Geom. 10 (1975), 113-134.

5. J. L. Kazdan, Prescribing the curvature of a Riemannian manifold, CBMS Regional Conf. Ser. in Math., no. 57, Amer. Math. Soc., Providence, RI, 1985; reprinted with corrections 1987.

6. T. Ouyang, On the positive solutions of semilinear equations $\Delta u+\lambda u+h u^{p}=0$ on compact manifolds. II, Indiana Univ. Math. J. 40 (1991), 1083-1141.

7. A. Rauzy, Courbure scalaire prescrite, C. R. Acad. Sci. Paris Sér. I Math. 316 (1993), 273-276.

8. __ Multiplicité pour un problème de courbure scalaire prescrite (à paraître).

9. J. L. Vazquez et L. Véron, Solutions positives d'équations elliptiques semi-linéaires sur des variétés riemanniennes compactes, C. R. Acad. Sci. Paris. Sér. I Math. 312 (1991), 811-815.

16 RUe des Saints Peres, Paris 75007, France

E-mail address: rauzy mathp6.jussieu.fr 